\title{
A Comparison between Transcutaneous and Total Serum Bilirubin in Healthy-term Greek Neonates with Clinical Jaundice
}

\author{
Charalambos Neocleous, Alkistis Adramerina, Stefanos Limnaios, \\ Symeon Symeonidis, Chrysoula Spanou, Marina Malakozi, \\ Evangelos Mpampalis \\ Department of Medical Pediatrics, General Hospital of Drama, Drama, Greece \\ Received October 6, 2013; Accepted April 4, 2014.
}

Key words: Neonatal jaundice - Transcutaneous bilirubin - BiliCheck device

\begin{abstract}
The accuracy of transcutaneous bilirubin meters has been assessed in newborns from various ethnic backgrounds. However, there are limited data on Greek newborns. Our study examined the accuracy of transcutaneous bilirubin measurements in clinically jaundiced healthy-term Greek newborns, using total serum bilirubin as the reference standard, in order to re-evaluate our local guidelines about neonatal jaundice. Clinically jaundiced newborns requiring total serum bilirubin level estimation were recruited prospectively. 368 pairs of total serum bilirubin/transcutaneous bilirubin measurements were taken in 222 newborns, using a direct spectrophotometric device and the BiliCheck device, respectively. The level of agreement between the obtained transcutaneous bilirubin and total serum bilirubin values was assessed. Our data were analysed using the Stata/SE 12.0 (StataCorp LP, USA) statistical programme. The mean ( \pm SD) TSB was $225.4 \pm 25.4 \mu \mathrm{mol} / \mathrm{l}$ and the mean $( \pm \mathrm{SD}) \mathrm{TcB}$ was $237.9 \pm 21.0 \mu \mathrm{mol} / \mathrm{l}$. The correlation between the values was poor (Pearson's correlation coefficient 0.439; Lin's concordance coefficient 0.377 [95\% Cl 0.301 to 0.453 ]; $\mathrm{P}<0.001$ ). The Bland-Altman analysis demonstrated that transcutaneous bilirubin measurements tended to overestimate the total serum bilirubin value (mean difference $12.5 \pm 24.9 \mu \mathrm{mol} / \mathrm{l})$, with wide $95 \%$ limits of agreement $(-36.2 \mu \mathrm{mol} / \mathrm{l}$ to $61.3 \mu \mathrm{mol} / \mathrm{l})$. Transcutaneous bilirubin values did not correlate well with total serum bilirubin values, being often imprecise in predicting the actual total serum bilirubin levels. This permits us to continue estimating total serum bilirubin in clinically jaundiced newborns according to our local guidelines, in order to safely decide the appropriate care plan.
\end{abstract}

Mailing Address: Charalambos Neocleous, MD., PhD., Department of Medical Pediatrics, General Hospital of Drama, Drama, Greece; e-mail: xneoc@yahoo.gr 


\section{Introduction}

Neonatal jaundice is an important problem during the first week of life. Except for routine screening for metabolism errors, measurement of serum bilirubin is the most frequent laboratory test in the good infant nursery. According to our local guidelines, total serum bilirubin (TSB) values have to be estimated in every newborn with yellow discoloration between the "nipple line and the umbilicus" or below, in order to safely decide a care plan. Measuring the TSB or the transcutaneous bilirubin (TcB) levels are both cited as acceptable ways to assess neonatal jaundice (Ip et al., 2004; Kolman et al., 2007). However, the determination of TSB levels is an invasive, stressful and time-consuming procedure. On the contrary, available TCB measurement devices offer a time-effective and non-invasive method to estimate serum bilirubin levels (Kolman et al., 2007). Our study examined the accuracy of TcB measurements in clinically jaundiced healthyterm Greek newborns, using TSB as the reference standard, in order to re-evaluate our local guidelines on this topic. A good correlation between TcB and TSB values in clinically jaundiced newborns would be very helpful to reduce heel-stick blood sampling and the possible complications, as well as cost and parents' anxiety.

\section{Material and Methods}

Study setting

The study was conducted during the period March 2010 to March 2012 in the Department of Medical Pediatrics in the General Hospital in Drama, Greece. The study was approved by the institutional review board of the hospital. The parents of all newborns enrolled in the study gave verbal informed consent.

\section{Subject eligibility}

A newborn was eligible for the study when: (1) the ethnicity was Greek, (2) the gestational age was over 37 weeks, (3) the birth weight was $>2500 \mathrm{~g}$, (4) the age at the time of measurement was 24-96 hours, and (5) the child was clinically jaundiced between the "nipple line and the umbilicus" (i.e. the lower chest).

Newborns with $\mathrm{Rh}$ or $\mathrm{ABO}$ isoimmunization, glucose-6-phosphate dehydrogenase deficiency, major congenital malformations, haemoglobinopathies, evidence of liver disease or infection and positive direct Coombs test were excluded from the study.

\section{TSB-TCB measurements}

Blood samples $(50 \mu \mathrm{l})$ for TSB measurements were collected using the heel-stick technique and the measurements were performed using a Unistat bilirubinometer (Leica Inc., Buffalo, NY) a direct spectrophotometric device with accuracy (bias) $\pm 5 \%$. All measurements were taken by skilled physicians, according to the manufacturer's instructions regarding calibration and quality controls. Special care was taken to avoid the exposure of the collected samples to light. A total 
of two TSB measurements were obtained from each newborn and these were averaged to obtain a single TSB measurement. This TSB value was used in the subsequent analysis. The spectrophotometry was used as a reference, as this is the technique used in daily routines and has better agreement with high-pressure liquid chromatography than any other technique (Raimondi et al., 2012).

Within 20 minutes of TSB determination, TcB was measured on the newborn's forehead, using the BiliCheck device (SpectRX, Norcross, GA), protected them from direct sunlight and avoiding areas with hair, bruises, nevi or other skin anomalies. The BiliCheck device is a hand-held bilirubinometer that measures $T c B$ levels using multi-wave length spectral reflectance analysis. All measurements were taken with the same BiliCheck device, which was calibrated before each measurement in accordance with the manufacturer's recommendations. The BiliCheck device displays a calculated average of five measurements for each bilirubin estimation.

All measurements were performed in the ambient morning light by skilled physicians, according to the manufacturer's instructions regarding calibration and quality controls.

\section{Statistical analysis}

The agreement between the TSB and TcB values was assessed using Pearson's correlation and Lin's concordance coefficients. These coefficients alone are only a poor indicator of the agreement between the two diagnostic tests, so a modified Bland-Altman technique was used to assess TCB and TSB variability. In this analysis, the difference between TcB and TSB was plotted against the average of TcB and $\mathrm{TSB}$, and displayed with $95 \%$ confidence limits $[\mathrm{Cl}]$ in order to compare the variability across a range of bilirubin results.

Specificity and sensitivity were estimated at two outcomes of interest (205 $\mu \mathrm{mol} / /$ and $240 \mu \mathrm{mol} / \mathrm{l})$ because are clinically important values in determining whether healthy-term newborns are ready for discharge home at the age of $24 \mathrm{~h}$ and $48 \mathrm{~h}$. Separate logistic regression models using $T c B$ as a predictor were created for each outcome, and sensitivity and specificity values were calculated. These were then plotted as receiver operating characteristic (ROC) curves. As the sensitivity and the specificity of a test increase, the ROC curve moves towards the upper left-hand corner of the plot, with a correspondingly higher AUC (area under curve). AUC values of 0.5 lack any diagnostic ability whereas AUC values of 1.0 correspond to a perfect screening test. Our data were analysed using Stata/SE 12.0 (StataCorp LP, USA) statistical programme.

\section{Results}

Subjects

A total of 368 determinations were obtained from 222 newborns (127 males, 95 females). The mean gestational age was 39 weeks and the mean birth weight 
Table 1 - Subjects and samples characteristics

\begin{tabular}{lc}
\hline Characteristics & Mean $( \pm \mathrm{SD})$ or $\mathrm{n}(\%)$ \\
\hline Male/female & $127(57.2) / 95(42.8)$ \\
Gestational age (week) & $39( \pm 1.5)$ \\
Birth weight $(\mathrm{g})$ & $3247( \pm 526)$ \\
TSB $(\mu \mathrm{mol} / \mathrm{l})$ & $225.4 \pm 25.4$ \\
TcB $(\mu \mathrm{mol} / \mathrm{l})$ & $237.9 \pm 21.0$ \\
Age at time of measurements (h) & $75( \pm 21)$ \\
TSB $>171 \mu \mathrm{mol} / \mathrm{l}$ & $368(100 \%)$ \\
TSB $>205 \mu \mathrm{mol} / /$ & $282(78.8 \%)$ \\
TSB $>240 \mu \mathrm{mol} / \mathrm{l}$ & $111(32.6 \%)$ \\
\hline
\end{tabular}

Table 2 - Accuracy and precision of transcutaneous bilirubin (TcB) measurement based on the total serum bilirubin (TSB) value

\begin{tabular}{lcccc}
\hline TSB values & $\begin{array}{c}\text { Measure- } \\
\text { ments }(n)\end{array}$ & $\begin{array}{c}\text { Pearson's correlation } \\
\text { coefficient }(r)\end{array}$ & $\begin{array}{c}\text { Lin's concordance } \\
\text { coefficient }(95 \% \mathrm{Cl})\end{array}$ & $\begin{array}{c}\text { Av. diff. } \\
\text { TcB-TSB }\end{array}$ \\
\hline All & 368 & 0.439 & $0.377(0.301-0.453)$ & 12.528 \\
TSB $\leq 240$ & 257 & 0.403 & $0.253(0.179-0.326)$ & 21.092 \\
$205<$ TSB $\leq 240$ & 171 & 0.166 & $0.090(0.009-0.172)$ & 14.690 \\
TSB $>240$ & 111 & 0.183 & $0.134(-0.001-0.269)$ & -7.302 \\
TSB $\leq 205$ & 86 & 0.290 & $0.071(0.017-0.125)$ & 33.822 \\
\hline
\end{tabular}

was $3247 \pm 526 \mathrm{~g}$. The mean age at the time that measurements were taken was 75 hours (Table 1 ).

The mean $( \pm S D) T S B$ was $225.4 \pm 25.4 \mu \mathrm{mol} / /$ with an interquartile range of $171 \mu \mathrm{mol} / \mathrm{l}$ to $278 \mu \mathrm{mol} / \mathrm{l}$. The mean $( \pm \mathrm{SD}) \mathrm{TcB}$ was $237.9 \pm 21.0 \mu \mathrm{mol} / \mathrm{l}$ with an interquartile range of $191 \mu \mathrm{mol} / /$ to $267 \mu \mathrm{mol} / \mathrm{l}$ (Table 1). $257 \mathrm{TSB}$ values were below $240 \mu \mathrm{mol} / \mathrm{l}$ and $111 \mathrm{TSB}$ values above $240 \mu \mathrm{mol} / /$. More specifically, 86 TSB values were in the range of 171-205 $\mu \mathrm{mol} / \mathrm{l}, 171 \mathrm{TSB}$ values were in the range of $205-240 \mu \mathrm{mol} / \mathrm{l}$ and $111 \mathrm{TSB}$ values were in the range of $240-278 \mu \mathrm{mol} / /$ (Table 2 ).

\section{Correlations}

The comparison of all $368 \mathrm{TcB}$ and TSB measurements demonstrated that TcB correlated poorly with TSB (Pearson's correlation coefficient 0.439; Lin's concordance coefficient 0.377 [ $95 \% \mathrm{Cl} 0.301$ to 0.453 ]; $\mathrm{P}<0.001$ ) (Table 2). The correlation between TcB and TSB is shown in Figure 1 in reference to the line of perfect concordance and reduced major axis. The slope $(\beta)$ of the regression plot is 0.828 , illustrating the poor correlation between TcB and TSB. Overall, $T c B$ overestimated TSB, as suggested by the big offset or $Y$ intercept of the regression plot $(Y$ intercept $=51.217)$ (Figure 1). Accuracy and precision of TcB measurements based on several ranges of TSB values are shown in Table 2. 


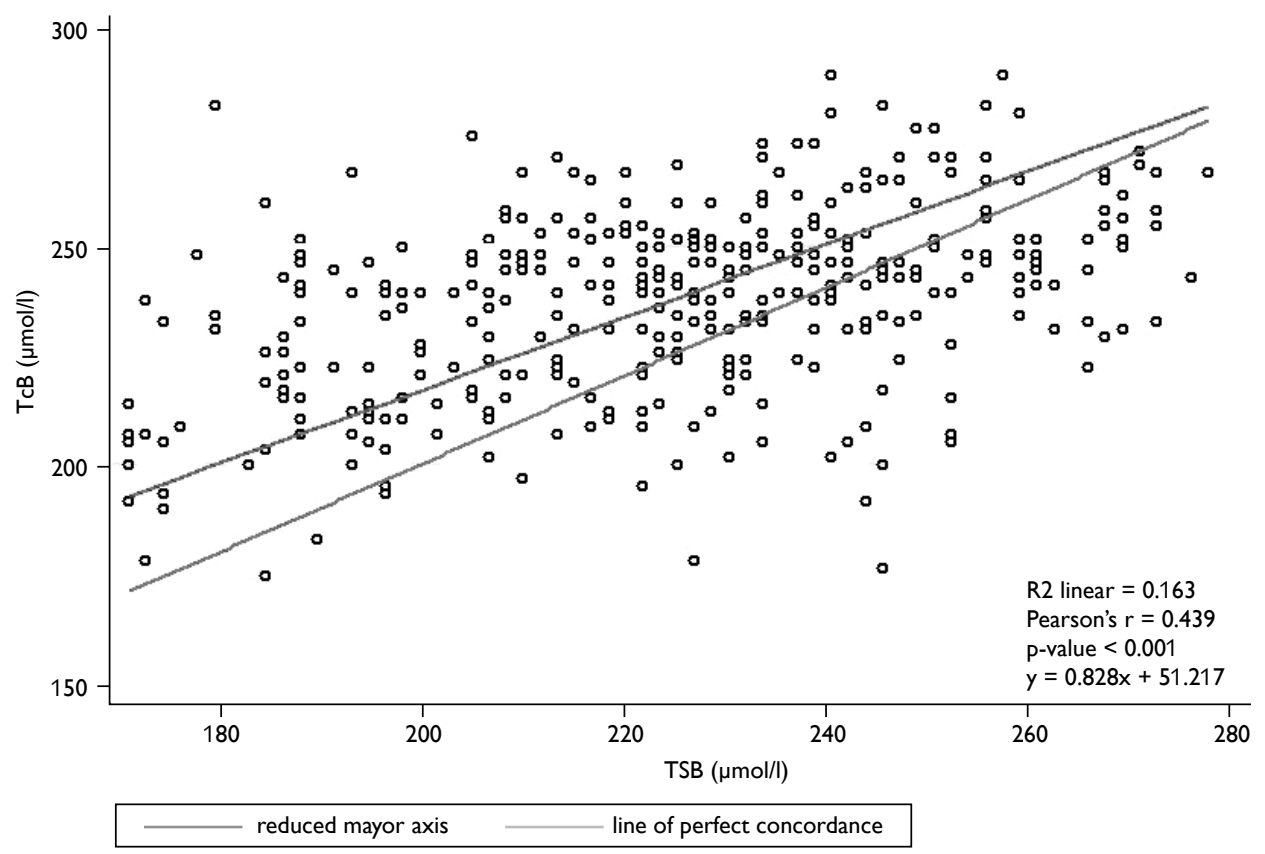

Figure 1 - Pearson's analysis.

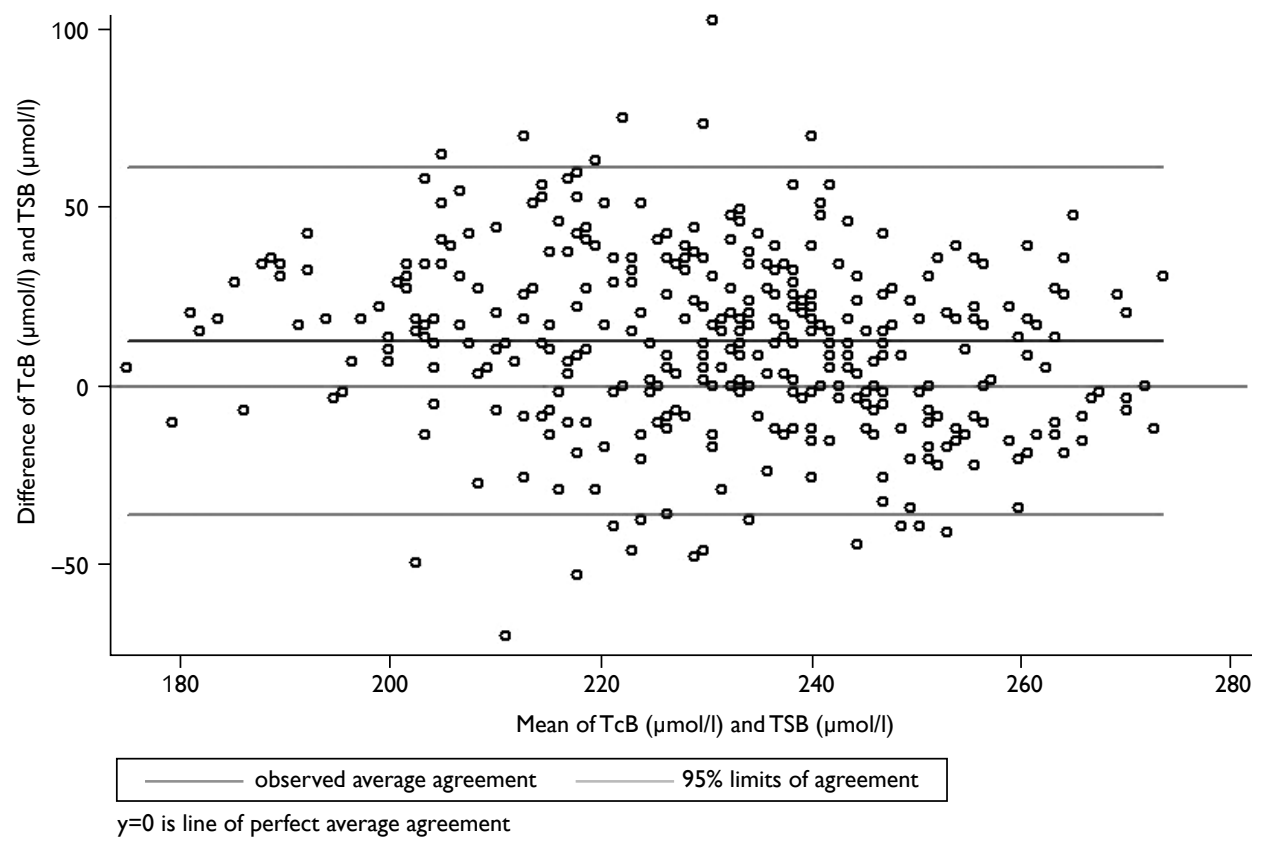

Figure 2 - Bland-Altman analysis. 
38) Prague Medical Report / Vol. 115 (2014) No. 1-2, p. 33-42

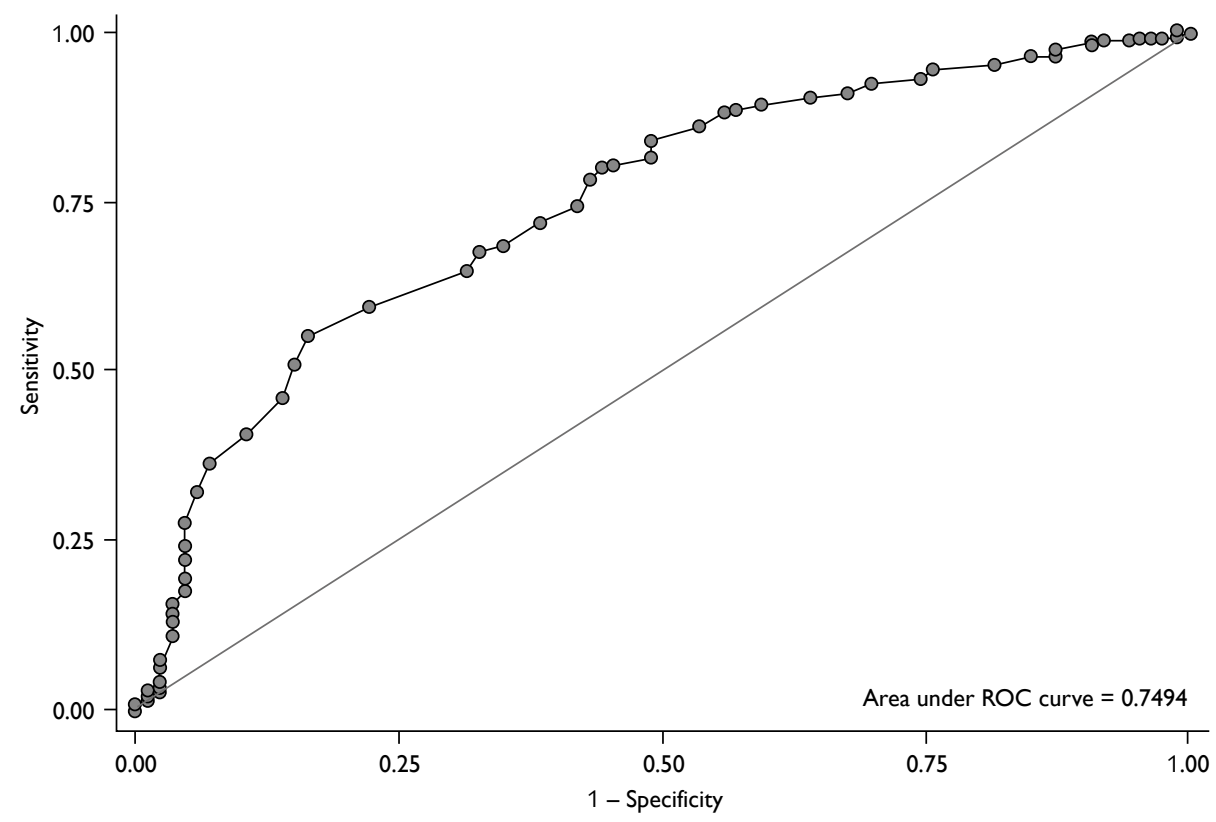

Figure $3-R O C$ curve for transcutaneous bilirubin values predicting a total serum bilirubin value of greater than $205 \mu \mathrm{mol} / \mathrm{l}$.

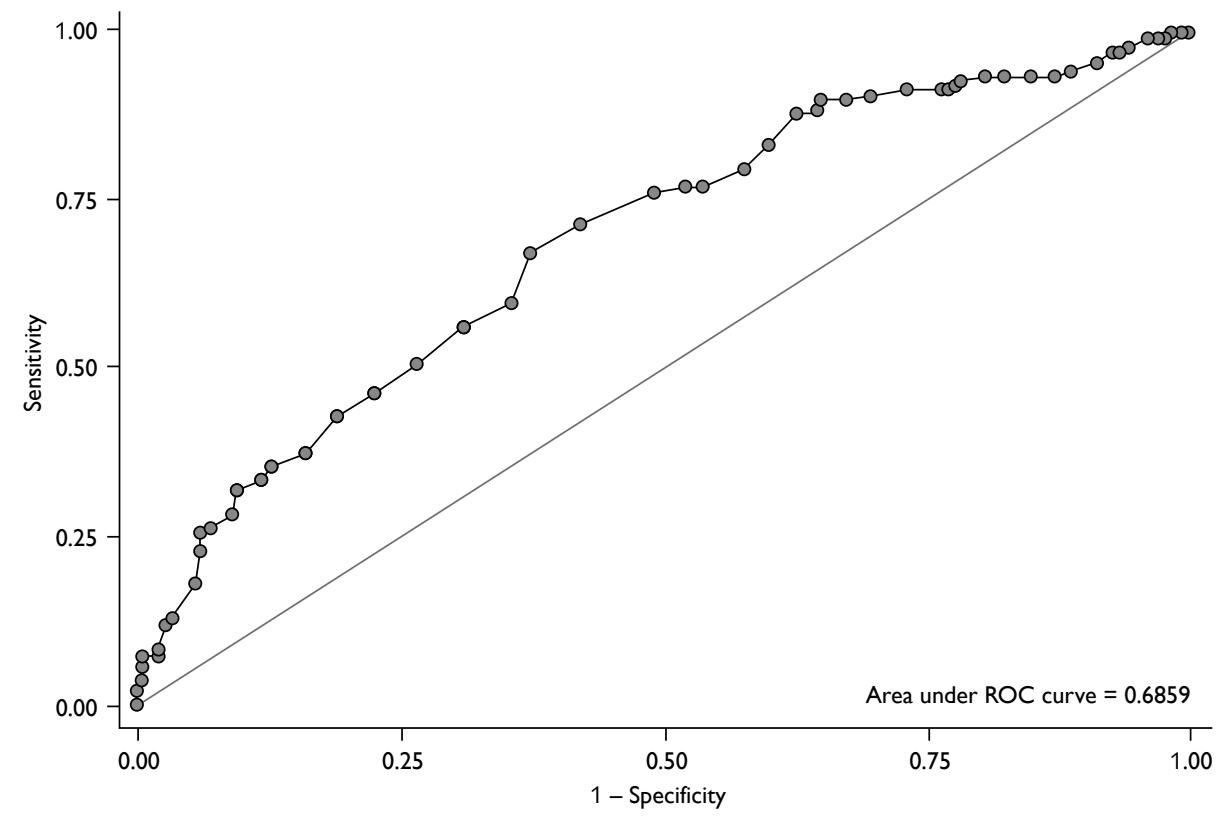

Figure $4-R O C$ curve for transcutaneous bilirubin values predicting a total serum bilirubin value of greater than $240 \mu \mathrm{moll} / \mathrm{l}$.

Neocleous Ch.; Adramerina A.; Limnaios S.; Symeonidis S.; Spanou Ch.; Malakozi M.; Mpampalis E. 
The Bland-Altman error plot demonstrates the TcB-TSB agreement of the BiliCheck device by comparing the difference versus the average of measurements between TCB and TSB values. The average error in evaluating hyperbilirubinaemia with TcB compared with evaluation with TSB (calculated by TcB-TSB) was $12.5 \pm 24.9 \mu \mathrm{mol} / \mathrm{l}(95 \%[\mathrm{Cl}]=-36.2 \mu \mathrm{mol} / \mathrm{l}$ to $61.3 \mu \mathrm{mol} / \mathrm{l})$. A Bland-Altman error plot illustrated that $259(70.38 \%)$ data points were positive; indicating that TcB measurements significantly overestimated TSB values (Figure 2).

Sensitivity analysis was performed for TSB values of interest at $24 \mathrm{~h}$ and $48 \mathrm{~h}$ of age, respectively. To detect a TSB value of $205 \mu \mathrm{mol} / \mathrm{l}$, a TcB value of $207 \mu \mathrm{mol} / \mathrm{l}$ would provide $95.4 \%$ sensitivity and $18.6 \%$ specificity (positive predictive value would be $79.4 \%$ and negative predictive value $55.2 \%$ ). Similarly, to detect a TSB value of $240 \mu \mathrm{mol} / \mathrm{l}$, a TcB of $265 \mu \mathrm{mol} / \mathrm{l}$ would provide $22.5 \%$ sensitivity and $94.2 \%$ specificity (positive predictive value would be $62.5 \%$ and negative predictive value 73.8\%). Refer to Figures 3 and 4, respectively, for graphical representations.

\section{Discussion}

The accuracy of TCB meters to estimate serum bilirubin levels has been assessed in newborns from various ethnic backgrounds (Slusher et al., 2004; Janjindamai and Tansantiwong, 2005; Kolman et al., 2007). However, to our knowledge, this is the first hospital-based study performed in Greece that has compared TcB and TSB measurements in healthy full-term clinically jaundiced Greek newborns.

It is noteworthy that our findings are not in concordance with previously performed studies. In our study, the Pearson's correlation coefficient was significantly lower compared to previous studies $[r=0.87$ to 0.96$]$ (Bhutani et al., 2000; Engle et al., 2002, 2005; Maisels et al., 2004; Slusher et al., 2004; Eggert et al., 2006; Jangaard et al., 2006; Boo and Ishak, 2007; Kolman et al., 2007; Karon et al., 2008; Campbell et al., 2011). Although the $95 \% \mathrm{Cl}(-36.213 \mu \mathrm{mol} / \mathrm{l}$ to $61.269 \mu \mathrm{mol} / \mathrm{l})$ for the TCB method were comparable to previous studies, they are too wide at the specific range of checked bilirubin values. Small differences between TcB and TSB measurements when bilirubin values ranged between 171 and $278 \mu \mathrm{mol} / \mathrm{l}$ are of great clinical importance, especially in healthy-term newborns at the age of $24 \mathrm{~h}$ to $72 \mathrm{~h}$, as even a small difference could change the care plan, according to the recent guidelines for the management of hyperbilirubinaemia in newborns (American Academy of Pediatrics Subcommittee on Hyperbilirubinemia, 2004).

Bland-Altman analysis demonstrated that most $T c B$ values $(n=259 ; 70.38 \%)$ overestimated TSB, while at high levels of jaundice they either underestimated or overestimated TSB values (Figure 2). Although it is unlikely to be clinically important, the overestimation of the TSB values could change care plans for jaundiced newborns. It is worth noting also that this may be per se protective against the clinical damage of hyperbilirubinaemia. On the other hand, in cases where TcB underestimates TSB, as happened at higher bilirubin levels, newborns requiring close follow-up or phototherapy might be missed. Consequently the BiliCheck device 
was often imprecise in predicting the actual TSB, and could not substitute the measurement of TSB in clinically jaundiced newborns.

Studies where TcB values correlated well with TSB values have been limited by the fact that TCB and TSB values were estimated in all newborns, i.e. as a routine screening rather than in clinically jaundiced newborns. Therefore, they demonstrated the accuracy and precision of TcB values to estimate TSB values at relatively low serum bilirubin levels, where TcB-measurement devices can provide a valid estimation of the TSB level, according to the American Academy of Pediatrics' guidelines for the management of hyperbilirubinemia in the newborn (AAP).TcB measurements have also tended to be taken by a handful of single users (i.e. research nurses or clinical investigators). However, in our study both TcB and TSB values were measured only in clinically jaundiced newborns and were obtained by a number of health care professionals. From our point of view, focusing on clinically jaundiced newborns and TCB measurements taken by a number of health care professionals represent more realistic practices in the health care setting, as in daily clinical practice this is the point at which follow-up or treatment decisions are made (Engle et al., 2002; Campbell et al., 2011).

There is one recent prospective cohort study where TcB values were measured in clinically jaundiced newborns and were found to correlate well $(r=0.83)$ with TSB values (Campbell et al., 2011). However, this was probably because the number of TSB measurements $<200 \mu \mathrm{mol} / \mathrm{l}$ was large $(n=266 ; 61.9 \%)$. On the contrary, TcB was found to correlate poorly with TSB > $200 \mu \mathrm{mol} / \mathrm{l}(\mathrm{n}=164$; $38 \%$ ) and TSB $>250 \mu \mathrm{mol} / \mathrm{l}(\mathrm{n}=68 ; 15.8 \%)$. It is worthy of note that in our study the Pearson correlation for TSB values between 171 and $278 \mu \mathrm{mol} / \mathrm{l}$ and also between $240 \mu \mathrm{mol} / \mathrm{l}$ and $278 \mu \mathrm{mol} / \mathrm{l}(\mathrm{r}=0.439$ and $\mathrm{r}=0.183$, respectively) were similar to those found by Campbell et al. (2011) at similar ranges of TSB values $200-267 \mu \mathrm{mol} / \mathrm{l}(r=0.52)$ and $250-267 \mu \mathrm{mol} / \mathrm{l}(r=0.23)$. Consequently, it seems that the major reason of the discrepancy between our findings and previous studies' findings is related to our relatively large number of TSB values $>240 \mu \mathrm{mol} / \mathrm{l}$ and significant smaller number of TSB values $<205 \mu \mathrm{mol} / \mathrm{l}$.

The results of Bhutani et al. (2000) and Campbell et al. (2011) are in concordance with our findings in that TcB values are more accurate at lower TSB values. However, in our study, Pearson correlation at TSB values below $240 \mu \mathrm{mol} / \mathrm{l}$ was still significantly lower. Probably, the reason is the narrow range of our TSB values $(171-240 \mu \mathrm{mol} / \mathrm{l})$. Previous studies included in their cohort a wider range of TSB values. Campbell et al. (2011) included 266 (62\%) TSB measurements between 99-200 $\mu \mathrm{mol} / / \mathrm{l}$ while in our study in the range 171-200 $\mu \mathrm{mol} / \mathrm{l}$ were included only 75 (20.3\%) TSB measurements.

Bhutani et al. (2000) used a TcB threshold of $220 \mu \mathrm{mol} / \mathrm{l}$ at the time of routine newborn screening (approximately $48 \mathrm{~h}$ of life) to perform further evaluation. Campbell et al. (2011) suggested an even lower TcB cut-off value to identify all significantly jaundiced newborns requiring phototherapy. To detect TSB values 
$>200 \mu \mathrm{mol} / \mathrm{l}$, they suggested a TcB threshold of $180 \mu \mathrm{mol} / \mathrm{l}$ when using the BiliCheck device, and to detect TSB values $>250 \mu \mathrm{mol} / \mathrm{l}$ they suggested a TcB cut-off value of $200 \mu \mathrm{mol} / \mathrm{l}$. In our cohort, for detecting TSB values of $205 \mu \mathrm{mol} / /$ or greater, we suggest a TcB threshold of $207 \mu \mathrm{mol} / \mathrm{l}$ when using the BiliCheck device in order to safely identify the newborns of age $24-72 \mathrm{~h}$ requiring close follow-up or phototherapy. When the clinician needs to identify TSB values $>240 \mu \mathrm{mol} / \mathrm{l}$, we suggest a TCB cut-off value of $265 \mu \mathrm{mol} / \mathrm{l}$ with this device. It is worthy of note that our TCB threshold values were higher than the checked TSB values, probably because TCB values tended to overestimate TSB values.

Nevertheless, when the BiliCheck device is the only available method for clinical jaundice's assessment (i.e. in private practice or primary health care centres), it is suggested that the TcB result should be summed with the $95 \% \mathrm{Cl}$ of the device, so as to estimate the maximum probable TSB concentration. Also, threshold values closer to the confidence limits would be appropriate to assist the clinical decisionmaking. In this way newborns requiring close follow-up or phototherapy would not be missed and unnecessary phototherapy or other treatment would be avoided.

It is our opinion that the most important limitation in our study was that the TSB values used in our cohort were not obtained using high-performance liquid chromatography, which is the gold standard for bilirubin measurement. Our hospital's capacities do not allow us to carry out this test in a large number of newborns. However, many laboratories in Greece use conventional TSB measuring techniques, which are routinely used in clinical decision-making.

\section{Conclusion}

In the present prospective cohort study regarding clinically jaundiced healthy-term newborns, TcB and TSB values did not correlate well and TCB values often proved imprecise in predicting actual TSB levels. These findings permit us to safely decide on a care plan for clinically jaundiced newborns using our present local guidelines. However, TcB measurement devices could be used in special instances if the limits of agreement between TSB and TcB levels are considered and a clinical judgement is maintained.

\section{References}

American Academy of Pediatrics Subcommittee on Hyperbilirubinemia (2004) Management of hyperbilirubinemia in the newborn infant 35 or more weeks of gestation. Pediatrics 114, 297-316.

Bhutani, V. K., Gourley, G. R., Adler, S., Kreamer, B., Dalin, C., Johnson, L. H. (2000) Nonivasive measurement of total serum bilirubin in a multiracial predischarge newborn population to assess the risk of severe hyperbilirubinemia. Pediatrics 106, E17.

Boo, N.Y., Ishak, S. (2007) Prediction of severe hyperbilirubinaemia using the Bilicheck transcutaneous bilirubinometer. J. Paediatr. Child Health 43, 297-302.

Campbell, D. M., Danayan, K. C., McGovern, V., Cheema, S., Stade, B., Sgro, M. (2011) Transcutaneous bilirubin measurement at the time of hospital discharge in a multiethnic newborn population. Paediatr. Child Health 16, 141-145. 
Eggert, L. D.,Wiedmeier, S. E., Wilson, J., Christensen, R. D. (2006) The effect of instituting a prehospital-discharge newborn bilirubin screening program in an 18-hospital health system. Pediatrics 117, e855-e862.

Engle, W. D., Jackson, G. L., Sendelbach, D., Manning, D., Frawley, W. H. (2002) Assessment of a transcutaneous device in the evaluation of neonatalhyperbilirubinemia in a primarily Hispanic population. Pediatrics $\mathbf{1 1 0}$, 61-67.

Engle, W. D., Jackson, G. L., Stehel, E. K., Sendelbach, D. M., Manning, M. D. (2005) Evaluation of a transcutaneous jaundice meter following hospital discharge in term and near-term neonates. J. Perinatol. 25, 486-490.

Ip, S., Chung, M., Kulig, J., O’Brien, R., Sege, R., Glicken, S., Maisels, M. J., Lau, J.; American Academy of Pediatrics Subcommittee on Hyperbilirubinemia (2004) An evidence-based review of important issues concerning neonatal hyperbilirubinemia. Pediatrics 114, e130-e153.

Jangaard, K., Curtis, H., Goldbloom, R. (2006) Estimation of bilirubin using BiliChektrade mark, a transcutaneous bilirubin measurement device: Effects of gestational age and use of phototherapy. Paediatr. Child Health 11, 79-83.

Janjindamai, W., Tansantiwong, T. (2005) Accuracy of transcutaneous bilirubinometer estimates using BiliCheck in Thai neonates. J. Med. Assoc. Thai. 88, 187-190.

Karon, B. S., Teske, A., Santrach, P. J., Cook, W. J. (2008) Evaluation of the BiliChek non-invasive bilirubin analyzer for prediction of serum bilirubin and risk of hyperbilirubinemia. Am. J. Clin. Pathol. 130, 976-982.

Kolman, K. B., Mathieson, K. M., Frias, C. (2007) A comparison of transcutaneous and total serum bilirubin in newborn Hispanic infants at 35 or more weeks of gestation. J. Am. Board Fam. Med. 20, 266-271.

Maisels, M. J., Ostrea, E. M., Touch, S., Clune, S. E., Cepeda, E., Kring, E., Gracey, K., Jackson, C., Talbot, D., Huang, R. (2004) Evaluation of a new transcutaneous bilirubinometer. Pediatrics 113, 1628-1635.

Raimondi, F., Lama, S., Landolfo, F., Sellitto, M., Borrelli, A. C., Maffucci, R., Milite, P., Capasso, L. (2012) Measuring transcutaneous bilirubin: a comparative analysis of three devices on a multiracial population. BMC Pediatr. 12, 70.

Slusher, T. M., Angyo, I. A., Bode-Thomas, F., Akor, F., Pam, S. D., Adetunji, A. A., McLaren, D.W., Wong, R. J., Vreman, H. J., Stevenson, D. K. (2004) Transcutaneous bilirubin measurements and serum total bilirubin levels in indigenous African infants. Pediatrics 113, 1636-1641. 